\section{Meaning of negations as a function of oppositional relationships*}

\author{
RAYMOND BAIRD \\ Wright State University, Dayton, Ohio 45431
}

Between-S agreement was used as a measure of the strength of the oppositional relationship holding between word pairs. This measure was found to be significantly correlated with between-S agreement regarding the affirmative meaning of negations of the form "not X."

In the study of meaning, a distinction is commonly made between negation and opposition (Deese, 1970; Lyons, 1963). Negation is the logical operation by which the complement of an event is specified. That is, asserting that an event is "not $X$ " implies that the event in question is from the residual sample space $\mathrm{S}-\mathrm{X}$. If " $\mathrm{X}$ " and "not $\mathrm{X}$ " are mutually exclusive and exhaustive binary events (e.g., heads and tails), then the negation "not heads" is equivalent to the assertion "tails." If, however, the class "not $\mathrm{X}$ " comprises more than one simple event (e.g., all integers except 7), a negation (e.g., "not 7") carries very little specific information.

Linguists argue, however, that in everyday language use oppositional relationships frequently determine the "implication" or meaning of negative information. That is, asserting that an event is "not $X$ " many times denotes the consensually held particular opposite of event "X." For example, strictly speaking, the statement "It is not raining" could mean that the weather was clear, snowing, foggy, sleeting, etc. However, "It is not raining" is frequently taken to mean "It is clear."

This study was designed to test the hypothesis that negative information of the form "not X" denotes a specific alternative when there exists a widely held consensually defined opposite to "X," even when there are several possible alternatives in the class "not $\mathrm{X}$." The study was conducted in three

*The assistance of Patricia Heuser and Mike Orico in completing this study is gratefully acknowledged. phases: (1) assessment of the strength of oppositional relationship existing between pairs of English words, (2) determination of the meaning or language forms, and (3) determination of the strength of the correlation between the two. It was predicted that strength of the oppositional relationship between word pairs would be positively correlated with the degree to which negation of one of the

In the first phase of the study, 44 common English words (including mass nouns, count nouns, verbs, and adjectives) were listed on sheets of paper. Fifteen college students were instructed to write beside each word the single English word each thought was most nearly opposite in meaning to the stimulus word. Each stimulus word and its most frequently given opposite comprised the word pairs. The strength of the oppositional relationship holding between the words of each pair was computed with a ratio of the number of Ss giving the most frequent response divided by the total number of responses given by all Ss.

From the 44 word pairs established in this way, 17 were chosen for use in the second phase of the study. (The number of word pairs was reduced because of limitations on the length of time each $S$ could participate.) The stimulus words of the 17 pairs were nouns and verbs. 1 The strengths of the oppositional relationships within these pairs ranged from .12 to .92 . Among these 17 pairs, there were none in which all Ss agreed on the best "opposite." Thus, for the sample of word pairs used here, " $\mathrm{X}$ " and "not $X$ " are not binary events-there are psychological implication of negative words in a pair implies the other. several alternatives in the class "not X" for each word pair. The number of alternative "opposites" given by Ss for a particular word ranged from two to eight. The number of alternatives in the oppositional class for each word was negatively correlated with the measure of inter-S agreement regarding the most frequently chosen opposite $(\mathrm{r}=-.71, \mathrm{p}<.005)$.

In the second phase of the study, 24 college students were shown stimulus items like

\section{He will not jump. He will}

Ss were asked to fill in the blank with the word the writer or speaker of the item would accept as being synonymous with "not jump." For each of the 17 stimulus sets, the proportion of students filling in the blank with the previously defined opposite was computed. This measure indicates the degree to which the negative phrase can be said to have "meaning" in the denotative sense.

The meaning agreement proportions were found to be significantly correlated with the strength of the oppositional relationships for the corresponding word pairs $(r=+.68$, $\mathrm{p}<.005)$. This finding supports the long-held hypothesis that, in ordinary language use, the psychological meaning of negative information is quite different from the logical meaning of the same information. Psychologically, negative information of the form "not $X$ " can denote a specific alternative, even when " $X$ " and "not X" are not exhaustive binary events, if there exists a widely held "opposite" to "X."

\section{REFERENCES}

DEESE, J. Psycholinguistics. Boston: Allyn \& Bacon, 1970.

LYONS, J. Structural semantics. Oxford, England: Philological Society (Basil Blackwell), 1963.

NOTE

1. The stimulus words are given first and the most frequently chosen opposite is given second; numbers in parenthesis are the proportions of Ss who agreed regarding the "best" opposite: come-go (.57), joy-sorrow (.21), salt-pepper (.64), lift-drop (.58), running-walking (.5), numbers-letters (.92), stand-sit (.69), buying-selling (.75), city-country (.57), moon-sun (.61), playing-working (.82), bread-water (.43), jump-fall (.33), carpet-floor (.12), eating-starving (.33), chair-table (.27), justice-injustice (.25). 